\title{
International Conference on Advances in Mobile Computing and Multimedia
}

\author{
Gabriele Kotsis • David Taniar • Ismail Khalil • \\ Eric Pardede
}

Published online: 17 September 2009

(C) Springer-Verlag 2009

This issue features selected papers from the International Conference on Advances in Mobile Computing and Multimedia (MoMM2008) held in Linz, Austria, in 24-26 November 2008. A number of important achievements marked the sixth edition of MoMM, including being endorsed by the ACM SIGMM and the proceedings is published by the ACM press making it available in the ACM Digital Library.

This year, Multimedia Systems Journal (MSJ) has agreed to publish the extension of MoMM2008 best papers appear in a special issue. A special thanks to MSJ Editor-in-chief, Professor Thomas Plagemann for this opportunity. We hope this current issue is a starting point of the future collaboration between MSJ and MoMM conference series.

Four papers are included in this issue. These papers have been extended from their original versions presented at the conference. The authors come from Canada, Saudi Arabia, Spain, Japan, Kuwait, Australia and Iran, which indicates the global audience of MoMM conference series and MSJ readers.

As general co-chairs and program co-chairs of MoMM2008, as well as the guest editors of MSJ special issue of MoMM2008, we would like to congratulate the authors of papers appeared in this issue for their achievements in MoMM2008 and their inclusion in this issue of MSJ. We would also like to thank the PC members of MoMM2008 who conducted the initial reviewing process for the conference and the MoMM2008 award co-chairs for selecting the best papers.

\footnotetext{
G. Kotsis · I. Khalil ( $\varangle)$

Institute of Telecooperation,

Johannes Kepler University Linz,

Altenberger Strasse 69, 4040 Linz, Austria

e-mail: Ismail.Khalil@jku.at; ismail@iiwas.org

G. Kotsis

e-mail: Gabriele.Kotsis@jku.at

D. Taniar

Clayton School of Information Technology,

Monash University, Clayton, VIC 3800, Australia

E. Pardede

Department of Computer Science and Computer Engineering,

La Trobe University, Bundoora, VIC 3083, Australia
} 